\title{
Eigenvalue of boundary value problem for nonlinear singular third-order $q$-difference equations
}

\author{
Changlong $\mathrm{Yu}^{*}$ and Jufang Wang
}

"Correspondence:

changlongyu@126.com

College of Sciences, Hebei

University of Science and

Technology, Shijiazhuang, Hebei 050018, P.R. China

\begin{abstract}
In this paper, we establish the existence of positive solutions of a boundary value problem for nonlinear singular third-order $q$-difference equations $D_{q}^{3} u(t)+\lambda a(t) f(u(t))=0, t \in I_{q}, u(0)=0, D_{q} u(0)=0, \alpha D_{q} u(1)+\beta D_{q}^{2} u(1)=0$, by using Krasnoselskii's fixed-point theorem on a cone, where $\lambda$ is a positive parameter. Finally, we give an example to demonstrate the use of the main result of this paper. The conclusions in this paper essentially extend and improve known results.
\end{abstract}

Keywords: q-difference equations; positive solutions; singular boundary value problem; Krasnoselskii's fixed-point theorem

\section{Introduction}

The $q$-difference equations initiated in the beginning of the 20th century [1-4], is a very interesting field in difference equations. In the last few decades, it has evolved into a multidisciplinary subject and plays an important role in several fields of physics, such as cosmic strings and black holes [5], conformal quantum mechanics [6], and nuclear and highenergy physics [7]. For some recent work on $q$-difference equations, we refer the reader to [8-12]. However, the theory of boundary value problems (BVPs) for nonlinear $q$-difference equations is still in an early stage and many aspects of this theory need to be explored. To the best of our knowledge, for the BVPs of nonlinear third-order $q$-difference equations, a few works were done, see $[13,14]$ and the references therein.

Recently, in [15], El-Shahed has studied the existence of positive solutions for the following nonlinear singular third-order BVP:

$$
\left\{\begin{array}{l}
u^{\prime \prime \prime}(t)+\lambda a(t) f(u(t))=0, \quad 0 \leq t \leq 1, \\
u(0)=u^{\prime}(0)=0, \quad \alpha u^{\prime}(1)+\beta u^{\prime \prime}(1)=0,
\end{array}\right.
$$

by Krasnoselskii's fixed-point theorem on a cone.

More recently, in [13] Ahmad has studied the existence of positive solutions for the following nonlinear BVP of third-order $q$-difference equations:

$$
\left\{\begin{array}{l}
D_{q}^{3} u(t)=f(t, u(t)), \quad 0 \leq t \leq 1, \\
u(0)=0, \quad D_{q} u(0)=0, \quad u(1)=0,
\end{array}\right.
$$

by Leray-Schauder degree theory and some standard fixed-point theorems.

\section{Springer}

@2014 Yu and Wang; licensee Springer. This is an Open Access article distributed under the terms of the Creative Commons Attribution License (http://creativecommons.org/licenses/by/2.0), which permits unrestricted use, distribution, and reproduction in any medium, provided the original work is properly cited. 
Motivated by the work above, in this paper, we will study the following BVP of nonlinear singular third-order $q$-difference equations:

$$
\left\{\begin{array}{l}
D_{q}^{3} u(t)+\lambda a(t) f(u(t))=0, \quad t \in I_{q}, \\
u(0)=0, \quad D_{q} u(0)=0, \quad \alpha D_{q} u(1)+\beta D_{q}^{2} u(1)=0,
\end{array}\right.
$$

where $\lambda>0$ is a positive parameter, $a:(0,1) \rightarrow[0, \infty)$ is continuous and $0<\int_{0}^{1} a(t) d_{q} t<$ $\infty, f$ is a continuous function, $I_{q}=\left\{q^{n}: n \in N\right\} \cup\{0,1\}, q \in(0,1)$ is a fixed constant, and $\alpha, \beta \geq 0, \alpha+\beta>0$.

Obviously, when $q \rightarrow 1^{-}$, BVP (1.1) reduces to the standard BVP in [15].

Throughout this paper, we always suppose the following conditions to hold:

(C 1$) f \in C([0,1],[0,+\infty))$;

$\left(\mathrm{C}_{2}\right) \alpha, \beta \geq 0, \alpha+\beta>0$ and $\frac{\alpha-\beta}{\alpha+\beta} \leq q$.

\section{Preliminary results}

In this section, firstly, let us recall some basic concepts of $q$-calculus $[16,17]$.

Definition 2.1 For $0<q<1$, we define the $q$-derivative of a real-value function $f$ as

$$
D_{q} f(t)=\frac{f(t)-f(q t)}{(1-q) t}, \quad t \in I_{q}-\{0\}, \quad D_{q} f(0)=\lim _{t \rightarrow 0} D_{q} f(t) .
$$

Note that $\lim _{q \rightarrow 1^{-}} D_{q} f(t)=f^{\prime}(t)$.

Definition 2.2 The higher-order $q$-derivatives are defined inductively as

$$
D_{q}^{0} f(t)=f(t), \quad D_{q}^{n} f(t)=D_{q} D_{q}^{n-1} f(t), \quad n \in N
$$

For example, $D_{q}\left(t^{k}\right)=[k]_{q} t^{k-1}$, where $k$ is a positive integer and the bracket $[k]_{q}=\left(q^{k}-\right.$ $1) /(q-1)$. In particular, $D_{q}\left(t^{2}\right)=(1+q) t$.

Definition 2.3 The $q$-integral of a function $f$ defined in the interval $[a, b]$ is given by

$$
\int_{a}^{x} f(t) d_{q} t:=\sum_{n=0}^{\infty} x(1-q) q^{n} f\left(x q^{n}\right)-a f\left(a q^{n}\right), \quad x \in[a, b]
$$

and for $a=0$, we denote

$$
I_{q} f(x)=\int_{0}^{x} f(t) d_{q} t=\sum_{n=0}^{\infty} x(1-q) q^{n} f\left(x q^{n}\right)
$$

then

$$
\int_{a}^{b} f(t) d_{q} t=\int_{0}^{b} f(t) d_{q} t-\int_{0}^{a} f(t) d_{q} t
$$

Similarly, we have

$$
I_{q}^{0} f(t)=f(t), \quad I_{q}^{n} f(t)=I_{q} I_{q}^{n-1} f(t), \quad n \in N .
$$


Observe that

$$
D_{q} I_{q} f(x)=f(x)
$$

and if $f$ is continuous at $x=0$, then $I_{q} D_{q} f(x)=f(x)-f(0)$.

In $q$-calculus, the product rule and integration by parts formula are

$$
\begin{aligned}
& D_{q}(g h)(t)=D_{q} g(t) h(t)+g(q t) D_{q} h(t), \\
& \int_{0}^{x} f(t) D_{q} g(t) d_{q} t=[f(t) g(t)]_{0}^{x}-\int_{0}^{x} D_{q} f(t) g(q t) d_{q} t .
\end{aligned}
$$

Remark 2.1 In the limit $q \rightarrow 1^{-}$, the above results correspond to their counterparts in standard calculus.

Definition 2.4 Let $E$ be a real Banach space. A nonempty closed convex set $P \subset E$ is called a cone if it satisfies the following two conditions:

(i) $x \in P, \lambda \geq 0$ implies $\lambda x \in P$;

(ii) $x \in P,-x \in P$ implies $x=0$.

Theorem 2.1 (Krasnoselskii) [18] Let $E$ be a Banach space and let $K \in E$ be a cone in E. Assume that $\Omega_{1}$ and $\Omega_{2}$ are open subsets of $E$ with $0 \in \Omega_{1}$ and $\bar{\Omega}_{1} \subset \Omega_{2}$. Let $T: K \cap\left(\bar{\Omega}_{2} \backslash \Omega_{1}\right) \rightarrow$ $K$ be a completely continuous operator. In addition, suppose either

$\left(\mathrm{H}_{1}\right) \quad\|T u\| \leq\|u\|, \forall u \in K \cap \partial \Omega_{1}$ and $\|T u\| \geq\|u\|, \forall u \in K \cap \partial \Omega_{2}$ or

$\left(\mathrm{H}_{2}\right)\|T u\| \leq\|u\|, \forall u \in K \cap \partial \Omega_{2}$ and $\|T u\| \geq\|u\|, \forall u \in K \cap \partial \Omega_{1}$

holds. Then $T$ has a fixed point in $K \cap\left(\bar{\Omega}_{2} \backslash \Omega_{1}\right)$.

Lemma 2.1 Let $y \in C[0,1]$, then the $B V P$

$$
\left\{\begin{array}{l}
D_{q}^{3} u(t)+y(t)=0, \quad t \in I_{q}, \\
u(0)=0, \quad D_{q} u(0)=0, \quad \alpha D_{q} u(1)+\beta D_{q}^{2} u(1)=0,
\end{array}\right.
$$

has a unique solution

$$
u(t)=\int_{0}^{1} G(t, s ; q) y(s) d_{q} s
$$

where

$$
G(t, s ; q)=\frac{1}{(1+q)(\alpha+\beta)} \begin{cases}\alpha t^{2}(1-q s)+\beta t^{2}-(t-q s)\left(t-q^{2} s\right)(\alpha+\beta), & 0 \leq s \leq t \leq 1 \\ \alpha t^{2}(1-q s)+\beta t^{2}, & 0 \leq t \leq s \leq 1\end{cases}
$$

Proof Integrate the $q$-difference equation from 0 to $t$, we get

$$
D_{q}^{2} u(t)=-\int_{0}^{t} y(s) d_{q} s+a_{2} .
$$

Integrate (2.4) from 0 to $t$, and change the order of integration, we have

$$
D_{q} u(t)=-\int_{0}^{t}(t-q s) y(s) d_{q} s+a_{2} t+a_{1} .
$$


Integrating (2.5) from 0 to $t$, and changing the order of integration, we obtain

$$
u(t)=-\int_{0}^{t}\left(\frac{t^{2}+q^{3} s^{2}}{1+q}-q t s\right) y(s) d_{q} s+\frac{a_{2}}{1+q} t^{2}+a_{1} t+a_{0},
$$

where $a_{2}, a_{1}, a_{0}$ are arbitrary constants. Using the boundary conditions $u(0)=0, D_{q} u(0)=$ $0, \alpha D_{q} u(1)+\beta D_{q}^{2} u(1)=0$ in (2.6), we find that $a_{0}=a_{1}=0$, and

$$
a_{2}=\frac{1}{\alpha+\beta}\left(\alpha \int_{0}^{1}(1-q s) y(s) d_{q} s+\beta \int_{0}^{1} y(s) d_{q} s\right) .
$$

Substituting the values of $a_{2}, a_{1}$, and $a_{0}$ in (2.6), we obtain

$$
\begin{aligned}
u(t)= & -\int_{0}^{t}\left(\frac{t^{2}+q^{3} s^{2}}{1+q}-q t s\right) y(s) d_{q} s \\
& +\frac{t^{2}}{(1+q)(\alpha+\beta)}\left(\alpha \int_{0}^{1}(1-q s) y(s) d_{q} s+\beta \int_{0}^{1} y(s) d_{q} s\right) \\
= & \int_{0}^{1} G(t, s ; q) y(s) d_{q} s,
\end{aligned}
$$

where

$$
G(t, s ; q)=\frac{1}{(1+q)(\alpha+\beta)} \begin{cases}\alpha t^{2}(1-q s)+\beta t^{2}-(t-q s)\left(t-q^{2} s\right)(\alpha+\beta), & 0 \leq s \leq t \leq 1 \\ \alpha t^{2}(1-q s)+\beta t^{2}, & 0 \leq t \leq s \leq 1\end{cases}
$$

This completes the proof.

Remark 2.2 For $q \rightarrow 1$, equation (2.6) takes the form

$$
u(t)=-\frac{1}{2} \int_{0}^{t}(t-s)^{2} y(s) d_{q} s+\frac{a_{2}}{2} t^{2}+a_{1} t+a_{0},
$$

which is the solution of a classical third-order ordinary differential equation $u^{\prime \prime \prime}(t)+y(t)=0$ and the associated form of Green's function for the classical case is

$$
G(t, s)=\frac{1}{2(\alpha+\beta)} \begin{cases}\alpha t^{2}(1-s)+\beta t^{2}-(t-s)^{2}(\alpha+\beta), & 0 \leq s \leq t \leq 1 \\ \alpha t^{2}(1-s)+\beta t^{2}, & 0 \leq t \leq s \leq 1\end{cases}
$$

It is obvious that, when $\left(\mathrm{C}_{2}\right)$ holds, $G(t, s ; q) \geq 0$, and $G(t, s ; q) \leq G(1, s ; q), 0 \leq t, s \leq 1$.

Lemma 2.2 Let $\left(C_{2}\right)$ hold, then $G(t, s ; q) \geq g(t) G(1, s ; q)$ for $0 \leq t, s \leq 1$, where $g(t)=$ $\frac{4 \beta}{5(\alpha+\beta)} t^{2}$.

Proof If $t \leq s$, then

$$
\begin{aligned}
\frac{G(t, s ; q)}{G(1, s ; q)} & =\frac{\frac{\alpha t^{2}(1-q s)}{(1+q)(\alpha+\beta)}+\frac{\beta t^{2}}{(1+q)(\alpha+\beta)}}{\frac{\alpha(1-q s)}{(1+q)(\alpha+\beta)}+\frac{\beta}{(1+q)(\alpha+\beta)}}=\frac{t^{2}-\frac{\alpha q s}{\alpha+\beta} t^{2}}{1-\frac{\alpha q s}{\alpha+\beta}} \\
& \geq t^{2}-\frac{\alpha}{\alpha+\beta} t^{2}=\frac{\beta}{\alpha+\beta} t^{2} \geq \frac{4 \beta}{5(\alpha+\beta)} t^{2} .
\end{aligned}
$$


If $t \geq s$, then

$$
\begin{aligned}
\frac{G(t, s ; q)}{G(1, s ; q)} & =\frac{\frac{\alpha t^{2}(1-q s)}{(1+q)(\alpha+\beta)}+\frac{\beta t^{2}}{(1+q)(\alpha+\beta)}-\frac{t^{2}+q^{3} s^{2}}{1+q}+q t s}{\frac{\alpha(1-q s)}{(1+q)(\alpha+\beta)}+\frac{\beta}{(1+q)(\alpha+\beta)}-\frac{1+q^{3} s^{2}}{1+q}+q s}=\frac{-\frac{\alpha q s}{\alpha+\beta} t^{2}-q^{3} s^{2}+(1+q) q t s}{-\frac{\alpha q s}{\alpha+\beta}-q^{3} s^{2}+(1+q) q s} \\
& \geq \frac{(1+q) q t^{2}-\frac{\alpha q s}{\alpha+\beta} t^{2}-q^{3} s^{2}}{(1+q) q s-q^{3} s^{2}}=\frac{(1+q) t^{2}-\frac{\alpha s}{\alpha+\beta} t^{2}-q^{2} s^{2}}{(1+q) s-q^{2} s^{2}} \\
& \geq \frac{t^{2}-\frac{\alpha}{\alpha+\beta} t^{2}}{1+q-q^{2}} \geq \frac{4 \beta}{5(\alpha+\beta)} t^{2} .
\end{aligned}
$$

The proof is complete.

We consider the Banach space $C_{q}=C\left(I_{q}, R\right)$ equipped with standard norm $\|u\|=$ $\sup \left\{|u(t)|, t \in I_{q}\right\}, u \in C_{q}$. Define a cone $P$ by

$$
P=\left\{u \in C_{q} \mid u(t) \geq 0, u(t) \geq g(t)\|u\|, t \in I_{q}\right\} .
$$

It is easy to see that if $u \in P$, then $\|u\|=u(1)$.

Define an integral operator $T: P \rightarrow C_{q}$ by

$$
T u(t)=\lambda \int_{0}^{1} G(t, s ; q) a(s) f(u(s)) d_{q} s, \quad t \in I_{q}, u \in P .
$$

Obviously, $T$ is well defined and $u \in P$ is a solution of BVP (1.1) if and only if $u$ is a fixed point of $T$.

Remark 2.3 By Lemma 2.2, we obtain, for $u \in P, T u(t) \geq 0$ on $I_{q}$ and

$$
\begin{aligned}
T u(t) & =\lambda \int_{0}^{1} G(t, s ; q) a(s) f(u(s)) d_{q} s \geq \lambda g(t) \int_{0}^{1} G(1, s ; q) a(s) f(u(s)) d_{q} s \\
& \geq \lambda g(t) \sup _{t \in I_{q}} \int_{0}^{1} G(t, s ; q) a(s) f(u(s)) d_{q} s=g(t)\|T u\| .
\end{aligned}
$$

Thus $T(P) \subset P$.

We adopt the following assumption:

$\left(C_{3}\right) a(t) \in C\left((0,1), R^{+}\right)$may be singular at $t=0,1,0<\int_{0}^{1} a(t) d_{q} t<+\infty$, and $0<\int_{0}^{1} G(1$, $s ; q) a(t) d_{q} t<+\infty$.

Lemma 2.3 Assume $\left(\mathrm{C}_{1}\right),\left(\mathrm{C}_{2}\right)$, and $\left(\mathrm{C}_{3}\right)$ hold, then $T: P \rightarrow P$ is completely continuous.

Proof Define the functions $a_{n}(t)$ for $n \geq 2$ by

$$
a_{n}(t)= \begin{cases}\inf \left\{a(t), a\left(\frac{1}{n}\right)\right\}, & 0 \leq t \leq \frac{1}{n} \\ a(t), & \frac{1}{n} \leq t \leq 1-\frac{1}{n}, \\ \inf \left\{a(t), a\left(1-\frac{1}{n}\right)\right\}, & 1-\frac{1}{n} \leq t \leq 1\end{cases}
$$


Next, for $n \geq 2$, we define the operator $T_{n}: P \rightarrow P$ by

$$
T_{n} u(t)=\lambda \int_{0}^{1} G(t, s ; q) a_{n}(s) f(u(s)) d_{q} s, \quad t \in I_{q}, u \in P .
$$

Obviously, $T_{n}$ is completely continuous on $P$ for any $n \geq 2$ by an application of the AscoliArzelá theorem. Denote $B_{K}=\{u \in P:\|u\| \leq K\}$. Then $T_{n}$ converges uniformly to $T$ as $n \rightarrow \infty$. In fact, for any $t \in I_{q}$, for each fixed $K>0$ and $u \in B_{K}$, from $\left(C_{1}\right)$, we obtain

$$
\begin{aligned}
\left|T_{n} u(t)-T u(t)\right|= & \left|\lambda \int_{0}^{1} G(t, s ; q)\left[a(s)-a_{n}(s)\right] f(u(s)) d_{q} s\right| \\
\leq & \lambda \int_{0}^{\frac{1}{n}} G(1, s ; q)\left|a(s)-a_{n}(s)\right| f(u(s)) d_{q} s \\
& +\lambda \int_{\frac{1}{n}}^{1-\frac{1}{n}} G(1, s ; q)\left|a(s)-a_{n}(s)\right| f(u(s)) d_{q} s \\
& +\lambda \int_{1-\frac{1}{n}}^{1} G(1, s ; q)\left|a(s)-a_{n}(s)\right| f(u(s)) d_{q} s \rightarrow 0 \quad(n \rightarrow \infty),
\end{aligned}
$$

where we have used the fact that $G(t, s ; q) \geq 0$, and $G(t, s ; q) \leq G(1, s ; q), 0 \leq t, s \leq 1$. Hence, $T_{n}$ converges uniformly to $T$ as $n \rightarrow \infty$, and therefore $T$ is completely continuous also. This completes the proof.

\section{Main results}

In this section, we will apply Krasnoselskii's fixed-point theorem to the eigenvalue problem (1.1). First, we define some important constants:

$$
\begin{aligned}
& A_{q}=\int_{0}^{1} G(1, s ; q) a(s) g(s) d_{q} s, \quad B_{q}=\int_{0}^{1} G(1, s ; q) a(s) d_{q} s, \\
& F_{0}=\lim _{u \rightarrow 0^{+}} \sup \frac{f(u)}{u}, \quad f_{0}=\lim _{u \rightarrow 0^{+}} \inf \frac{f(u)}{u}, \\
& F_{\infty}=\lim _{u \rightarrow+\infty} \sup \frac{f(u)}{u}, \quad f_{\infty}=\lim _{u \rightarrow+\infty} \inf \frac{f(u)}{u} .
\end{aligned}
$$

Here we assume that $\frac{1}{A_{q} f_{\infty}}=0$ if $f_{\infty}=\infty$ and $\frac{1}{B_{q} F_{0}}=\infty$ if $F_{0}=0$ and $\frac{1}{A_{q} f_{0}}=0$ if $f_{0}=\infty$ and $\frac{1}{B_{q} F_{\infty}}=\infty$ if $F_{\infty}=0$.

The main result of this paper is the following.

Theorem 3.1 Suppose that $\left(\mathrm{C}_{1}\right),\left(\mathrm{C}_{2}\right)$ and $\left(\mathrm{C}_{3}\right)$ hold and $A_{q} f_{\infty}>B_{q} F_{0}$. Then for each $\lambda \in$ $\left(\frac{1}{A_{q} f_{\infty}}, \frac{1}{B_{q} F_{0}}\right), B V P(1.1)$ has at least one positive solution.

Proof By the definition of $F_{0}$, we see that there exists an $l_{1}>0$, such that $f(u) \leq\left(F_{0}+\varepsilon\right) u$ for $0 \leq u \leq l_{1}$. If $u \in P$ with $\|u\|=l_{1}$, we have

$$
\|T u\|=T u(1)=\lambda \int_{0}^{1} G(1, s ; q) a(s) f(u(s)) d_{q} s \leq \lambda\left(F_{0}+\varepsilon\right)\|u\| B_{q} .
$$

Choose $\varepsilon>0$ sufficiently small such that $\lambda\left(F_{0}+\varepsilon\right) B_{q} \leq 1$. Then we obtain $\|T u\| \leq\|u\|$. Thus if we let $\Omega_{1}=\left\{u \in C_{q} \mid\|u\|<l_{1}\right\}$, then $\|T u\| \leq\|u\|$ for $u \in P \cap \partial \Omega_{1}$. 
From the definition of $f_{\infty}$, we see that there exist an $l_{3}>0$ and $l_{3}>l_{1}$, such that $f(u) \geq$ $\left(f_{\infty}-\varepsilon\right) u$ for $u>l_{2}$. Let $l_{2}>l_{3}$, if $u \in P$ with $\|u\|=l_{2}$ we have

$$
\begin{aligned}
\|T u\| & =\operatorname{Tu}(1)=\lambda \int_{0}^{1} G(1, s ; q) a(s) f(u(s)) d_{q} s \\
& \geq \lambda \int_{0}^{1} G(1, s ; q) a(s) g(s) f(u(s)) d_{q} s \geq \lambda\left(f_{\infty}-\varepsilon\right)\|u\| A_{q} .
\end{aligned}
$$

Choose $\varepsilon>0$ sufficiently small such that $\lambda\left(f_{\infty}-\varepsilon\right) A_{q} \geq 1$. Then we have $\|T u\| \geq\|u\|$. Let $\Omega_{2}=\left\{u \in C_{q} \mid\|u\|<l_{2}\right\}$, then $\Omega_{1} \subset \bar{\Omega}_{2}$ and $\|T u\| \geq\|u\|$ for $u \in P \cap \partial \Omega_{2}$.

Condition $\left(\mathrm{H}_{1}\right)$ of Krasnoselskii's fixed-point theorem is satisfied. Hence, by Theorem 2.1, the result of Theorem 3.1 holds. This completes the proof of Theorem 3.1.

Theorem 3.2 Suppose that $\left(\mathrm{C}_{1}\right),\left(\mathrm{C}_{2}\right)$ and $\left(\mathrm{C}_{3}\right)$ hold and $A_{q} f_{0}>B_{q} F_{\infty}$. Then for each $\lambda \in$ $\left(\frac{1}{A_{q} f_{0}}, \frac{1}{B_{q} F_{\infty}}\right), B V P(1.1)$ has at least one positive solution.

Proof It is similar to the proof of Theorem 3.1.

Theorem 3.3 Suppose that $\left(\mathrm{C}_{1}\right),\left(\mathrm{C}_{2}\right)$ and $\left(\mathrm{C}_{3}\right)$ hold and $\lambda B_{q} f(u)<u$ for $u \in(0,+\infty)$. Then $B V P(1.1)$ has no positive solution.

Proof Assume to the contrary that $u$ is a positive solution of BVP (1.1). Then

$$
\begin{aligned}
u(1) & =\lambda \int_{0}^{1} G(1, s ; q) a(s) f(u(s)) d_{q} s<\frac{1}{B_{q}} \int_{0}^{1} G(1, s ; q) a(s) u(s) d_{q} s \\
& \leq \frac{u(1)}{B_{q}} \int_{0}^{1} G(1, s ; q) a(s) d_{q} s=u(1) .
\end{aligned}
$$

This is a contradiction and completes the proof.

Theorem 3.4 Suppose that $\left(C_{1}\right),\left(C_{2}\right)$ and $\left(C_{3}\right)$ hold and $\lambda A_{q} f(u)>u$ for $u \in(0,+\infty)$. Then $B V P(1.1)$ has no positive solution.

Proof It is similar to the proof of Theorem 3.3.

\section{Example}

Consider the following BVP:

$$
\left\{\begin{array}{l}
D_{\frac{1}{2}}^{3} u(t)+\lambda t^{-\frac{1}{2}} \frac{10 u^{2}+u}{u+1}(5+\sin u)=0, \quad t \in I_{q}, \\
u(0)=0, \quad D_{\frac{1}{2}} u(0)=0, \quad D_{\frac{1}{2}} u(1)+3 D_{\frac{1}{2}}^{2} u(1)=0 .
\end{array}\right.
$$

Then $F_{0}=6, f_{0}=4, F_{\infty}=60, f_{\infty}=40$, and $4 u \leq f(u) \leq 60 u$. By direct calculations, we obtain $A_{q}=0.110963$ and $B_{q}=0.271661$. From Theorem 3.1 we see that if $\lambda \in$ $(0.225299,0.613510)$ then the problem $(4.1)$ has a positive solution. From Theorem 3.3 we see that if $\lambda<0.061351$ then the problem (4.1) has no positive solution. By Theorem 3.4 we see that if $\lambda>2.252986$ then the problem (4.1) has no positive solution. 
Authors' contributions

Each of the authors, CY and JW contributed to each part of this work equally and read and approved the final version of the manuscript.

\section{Acknowledgements}

This work was supported by the Natural Science Foundation of China (10901045), (11201112) and (61304106), the Natural Science Foundation of Hebei Province (A2013208147) and (A2011208012).

\section{Received: 4 October 2013 Accepted: 26 December 2013 Published: 16 Jan 2014}

\section{References}

1. Jackson, FH: On q-difference equations. Am. J. Math. 32, 305-314 (1910)

2. Carmichael, RD: The general theory of linear q-difference equations. Am. J. Math. 34, 147-168 (1912)

3. Mason, TE: On properties of the solutions of linear $q$-difference equations with entire function coefficients. Am. J. Math. 37, 439-444 (1915)

4. Adams, CR: On the linear ordinary q-difference equation. Ann. Math. 30, 195-205 (1928)

5. Strominger, A: Information in black hole radiation. Phys. Rev. Lett. 71, 3743-3746 (1993)

6. Youm, D: q-Deformed conformal quantum mechanics. Phys. Rev. D 62, 095009 (2000)

7. Lavagno, A, Swamy, PN: q-Deformed structures and nonextensive statistics: a comparative study. Physica A 305(1-2), 310-315 (2002). Non extensive thermodynamics and physical applications (Villasimius, 2001)

8. Ahmad, B, Ntouyas, SK: Boundary value problems for q-difference inclusions. Abstr. Appl. Anal. 2011, Article ID $292860(2011)$

9. Dobrogowska, A, Odzijewicz, A: Second order q-difference equations solvable by factorization method. J. Comput. Appl. Math. 193, 319-346 (2006)

10. Ahmad, B: A study of second-order q-difference equations with boundary conditions. Adv. Differ. Equ. 2012,35 (2012). doi:10.1186/1687-1847-2012-35

11. El-Shahed, M, Hassan, HA: Positive solutions of q-difference equation. Proc. Am. Math. Soc. 138, 1733-1738 (2010)

12. $\mathrm{Yu}, \mathrm{CL}$, Wang, JF: Existence of solutions for nonlinear second-order q-difference equations with first-order q-derivatives. Adv. Differ. Equ. 2013, 124 (2013)

13. Ahmad, B: Boundary value problems for nonlinear third-order q-difference equations. Electron. J. Differ. Equ. 2011, 94 (2011)

14. Ahmad, B, Nieto, J: On nonlocal boundary value problem of nonlinear q-difference equations. Adv. Differ. Equ. 2012 81 (2012). doi:10.1186/1687-1847-2012-81

15. El-Shahed, M: Positive solutions for nonlinear singular third order boundary value problem. Commun. Nonlinear Sci. Numer. Simul. 14, 424-429 (2009)

16. Gasper, G, Rahman, M: Basic Hypergeometric Series. Cambridge University Press, Cambridge (1990)

17. Kac, V, Cheung, P: Quantum Calculus. Springer, New York (2002)

18. Guo, D, Lakshmikantham, V: Nonlinear Problems in Abstract Cones. Academic Press, San Diego (1988)

10.1186/1687-1847-2014-21

Cite this article as: Yu and Wang: Eigenvalue of boundary value problem for nonlinear singular third-order q-difference equations. Advances in Difference Equations 2014, 2014:21

\section{Submit your manuscript to a SpringerOpen ${ }^{\circ}$ journal and benefit from:}

- Convenient online submission

- Rigorous peer review

- Immediate publication on acceptance

- Open access: articles freely available online

- High visibility within the field

- Retaining the copyright to your article 